\title{
Evolution of atomic motion in an intense standing wave
}

\author{
Jian Chen, J. G. Story, and Randall G. Hulet \\ Physics Department and Rice Quantum Institute, Rice University, Houston, Texas 77251-1892
}

(Received 3 August 1992)

\begin{abstract}
We have investigated the effect of the dipole force and its fluctuation on the motion of $\mathrm{Li}$ atoms in an intense, one-dimensional, near-resonant standing light wave. The duration of the interaction of the atoms with the standing wave was varied from several tens of spontaneous-emission lifetimes to several hundreds. For a standing-wave frequency blue detuned from resonance, diffusive heating can dominate the time-averaged dissipative dipole force so that there is no steady-state momentum distribution. However, for sufficiently large blue detunings, the rate of diffusion is so slow that the resulting distribution approaches a quasisteady state. For red detunings, the diffusion is balanced with the force and a true steady state is achieved. We apply a Monte Carlo method based on the density-matrix equations in the dressed-state representation to simulate the atomic motion. The dynamics of atom channeling is discussed.
\end{abstract}

PACS number(s): $32.80 . \mathrm{Pj}, 42.50 . \mathrm{Vk}$

\section{INTRODUCTION}

Laser cooling of "two-level" atoms in the low-lightintensity regime known as Doppler cooling, is now well understood [1,2]. In this case, atoms move in light waves whose frequencies are tuned below the resonance frequency of an atomic transition and whose intensities are less than or near the saturation intensity of the transition. Theory accurately describes the dynamics of atomic motion for these conditions, and ultimate cooling temperatures can be precisely predicted [1,2]. In the case of a two-level atom moving in an intense standing wave, for which the light intensity is much greater than the saturation intensity of the cooling transition, the force on the atom is dominated by several fundamentally different physical mechanisms depending on the velocity of the atom and the intensity of the field [3]. Figure 1 shows the results of a calculation of the force on the atom averaged over the time for an atom to travel one optical wavelength, as a function of atomic momentum. It is assumed that the atom interacts with the field for a long time compared to the spontaneous emission lifetime of the excited state. This calculation employs a continuedfraction method to obtain the steady-state solution of the optical Bloch equations [4,5]. The parameters for the results shown in Fig. 1 are for an on-resonance Rabi frequency $\Omega_{0}=50 \gamma$ and a detuning $\Delta=+5 \gamma$, where $\hbar \Omega_{0}$ is the interaction energy of the atom with the field at an antinode, $\gamma$ is the spontaneous decay rate of the excited state, and $\Delta=\omega_{L}-\omega_{0}\left(\omega_{L}\right.$ is the laser frequency and $\omega_{0}$ is the atomic resonance frequency). The force on a slowly moving atom is due to the interaction of the induced atomic dipole moment with the spatial gradient of the field [3-7]. This "dipole force" is negative (i.e., cooling) for blue detuning (i.e., $\Delta>0$ ), and for atomic momentum smaller than a "critical momentum" $p_{c}$, as shown in Fig. 1. For the parameters used for Fig. $1, p_{c}=25 \hbar k$. The structure appearing at atomic momenta $p>p_{c}$ is due to velocity-resonant, multiphoton "doppleron" resonances
$[8,9]$. The doppleron resonances can be thought of as multiphoton Doppler cooling events involving $2 n+1$ photons $(n=0,1,2, \ldots)$. The force due to the dopplerons is heating for blue detuning.

Since the dipole force does not saturate at high intensity, it is potentially more efficient for slowing atoms than Doppler cooling, which does saturate [10-13]. However, the dipole force can have large fluctuations [3,5-7]. These fluctuations, which lead to momentum diffusion, are predominately caused by the randomness in the direc-

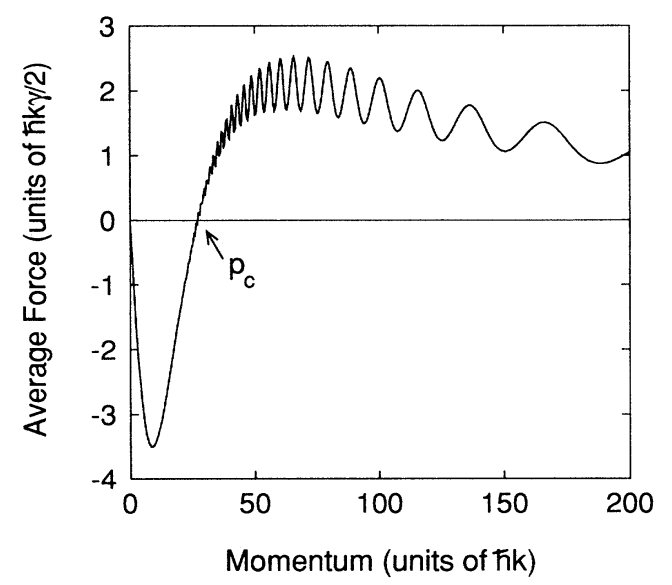

FIG. 1. The time-averaged force on an atom in a standing wave as a function of the momentum of the atom calculated by the continued-fraction method. The Rabi frequency is $\Omega_{0}=50 \gamma$, and the detuning of the standing-wave frequency from resonance is $\Delta=+5 \gamma$, where $\gamma$ is the radiative-decay lifetime. The force is given in units of the maximum of the one-photon Doppler force $\hbar k \gamma / 2$, and the momentum in units of the photon momentum $\hbar k$. In this case, the dipole force is negative (i.e., cooling) for blue detuning (i.e., $\Delta>0$ ), and for atomic momentum smaller than a "critical momentum" $p_{c}(25 \hbar k)$. The structure appearing at momenta higher than $p_{c}$ is due to the velocity-resonant, multiphoton "doppleron" resonances. 
tion of momentum recoil from stimulated transitions for a high-intensity standing wave. An atom can absorb a photon from either counterpropagating traveling-wave component of the standing wave and likewise, can be stimulated to emit a photon by either wave. As a result, the atom will random walk in momentum space, similar to the Brownian motion of a particle which random walks in real space as the result of collisions with surrounding particles. Spontaneous emission of photons in random directions can also cause the atomic momentum to fluctuate. However, the dominant fluctuation mechanism in an intense standing wave is due to stimulated processes since the stimulated transition rate is proportional to $\Omega_{0}^{2} / \gamma$, which can be much greater than $\gamma$. Like the time-averaged force, the rate of the diffusion due to stimulated processes does not saturate at high intensity. The ultimate cooling limit, where it exists, is determined by the balance of the time-averaged force with diffusion.

The subject of atomic motion in an intense standing wave has been investigated for various interaction times and laser parameter regimes [14]. Pritchard and associates, in a series of publications, studied the diffractive regime for which the interaction time $t<<\tau\left(\tau=\gamma^{-1}\right)$ [15], and the transition to the diffusive regime for which $t \sim \tau$ [16]. Arimondo, Lew, and Oka observed the diffusive spreading of the atomic velocity distribution for longer interaction times $t \gg \tau$ [17]. Localization of atoms in the optical potential wells of a standing wave was observed [18] and recently exploited to cool atoms adiabatically [19].

Various theoretical methods have been used to describe the evolution of atomic motion for $t \gg \tau$. One of the first of these methods used the Fokker-Planck equation (FPE) for the Wigner distribution [2]. However, since the FPE treats the motion of the atom classically it is not valid for $p \sim \hbar k$ (photon momentum). In addition, the usual approach is to average over the spatial variation of the atom's kinetic energy in the standing wave, so atom channeling (see Sec. IV) is not accounted for. A dressedstate Monte Carlo method, similar to that used in this paper, was developed by Dalibard et al., which is appropriate for $p<p_{c}$ [20]. Although this method also treats the atomic motion classically, it accounts for atom channeling and, as will be shown below, this method accurately models the atomic motional dynamics in a regime where the FPE approach fails. The "momentum family" approach developed by Castin, Wallis, and Dalibard is a fully-quantum-mechanical theory, and has been employed to successfully explain "Sisyphus" sub-Doppler cooling [21]. Unfortunately, this approach is not computationally feasible for the high laser intensity $\left(\Omega_{0} \gg \gamma\right)$ and small laser detuning $\left(\Delta<<\Omega_{0}\right)$ regime since an extremely large number of momentum families are coherently coupled in this case. However, the Monte Carlo wave-function (MCWF) approach recently developed by Dalibard, Castin, and Mølmer and by Dum, Zoller, and Ritsch is likely to be a very effective method for the high-intensity, fully-quantum-mechanical problem [22]. The main advantage of the MCWF method is that the number of coupled equations is of order $N$ in this wave-function description, while it is of order $N^{2}$ for the density-matrix-based momentum family method. For a typical situation discussed in this paper, $\Omega_{0}=50 \gamma$ and $\Delta<<\Omega_{0}, 10^{5}$ first-order differential equations are coupled in the momentum family approach, while the MCWF method requires only $\left(10^{5}\right)^{1 / 2} \sim 300$ coupled equations.

In this paper, we present experimental results and Monte Carlo simulations of the evolution of atomic motion in an intense, one-dimensional standing wave for the relatively poorly investigated regime corresponding to interaction times $t \gg \tau$ and for atomic momenta $p \sim p_{c}$. The paper is organized as follows. Section II describes the Monte Carlo method, based on the densitymatrix equations in the dressed-state basis. In Sec. III, the experiment is described and the experimental data are compared with the Monte Carlo results. In Sec. IV, the initial heating due to the sudden application of the standing wave is discussed, the dynamics of channeling is studied, and the results of calculations using the FokkerPlanck equation are compared with the data.

\section{MONTE CARLO METHOD IN THE DRESSED-STATE BASIS}

For an atom in a one-dimensional, single-mode standing light wave, the total Hamiltonian of the atom plus field is

$$
H=\frac{p^{2}}{2 M}+H_{A}+\hbar \omega_{L} \widehat{a}^{\dagger} \widehat{a}+V,
$$

where $p^{2} / 2 M$ is the kinetic energy of the atom, $H_{A}$ represents the internal atomic Hamiltonian, $\hbar \omega_{L} \widehat{a}^{\dagger} \widehat{a}$ is the energy of the quantized radiation field ( $\hat{a}^{\dagger}$ and $\hat{a}$ are the creation and destruction operators, respectively), and $V=-\mathbf{d} \cdot\left(\boldsymbol{\epsilon}_{L} \hat{a}+\boldsymbol{\epsilon}_{L}^{*} \hat{a}^{\dagger}\right)$ is the atom-field interaction energy in the rotating-wave approximation, where $d$ is the electric dipole moment of the atom. The quantized electric field is $\epsilon_{L}=i E_{0} \sin k z \widehat{\mathbf{e}} / n^{1 / 2}$, where $E_{0}$ is the maximum field strength of one traveling-wave component, $k$ is the wave vector which is along the $z$ axis, and $n$ is the number of photons in the mode with polarization $\widehat{\mathbf{e}}$.

By diagonalizing $H$ in the representation $\{|e, n\rangle,|g, n+1\rangle\}$, where $|e, n\rangle$ represents the state in which the atom is in the excited state with $n$ photons in the radiation field, and $|g, n+1\rangle$ represents the state in which the atom is in the ground state with $n+1$ photons, the eigenenergies are obtained [7]

$$
\begin{aligned}
& E_{1 n}(z)=\frac{p^{2}}{2 M}+(n+1) \hbar \omega_{L}-\frac{\hbar \Delta}{2}+\frac{\hbar \Omega}{2}, \\
& E_{2 n}(z)=\frac{p^{2}}{2 M}+(n+1) \hbar \omega_{L}-\frac{\hbar \Delta}{2}-\frac{\hbar \Omega}{2},
\end{aligned}
$$

where $\Omega \equiv \Omega(z)=\left[\Omega_{0}^{2} \sin ^{2} k z+\Delta^{2}\right]^{1 / 2}$. Treating the atomic motion classically, the corresponding eigenvectors (dressed states) are [7]

$$
\begin{aligned}
& |1, n\rangle=e^{i(\pi / 4)} \cos \theta|e, n\rangle+e^{-i(\pi / 4)} \sin \theta|g, n+1\rangle, \\
& |2, n\rangle=-e^{i(\pi / 4)} \sin \theta|e, n\rangle+e^{-i(\pi / 4)} \cos \theta|g, n+1\rangle,
\end{aligned}
$$




$$
\cos 2 \theta=-\frac{\Delta}{\Omega}, \quad \sin 2 \theta=\frac{\Omega_{0} \sin k z}{\Omega} .
$$

$\Omega_{0}$ is the resonant Rabi frequency defined as $\Omega_{0}=-2 \mathbf{d}_{e g} \cdot E_{0} \widehat{\mathbf{e}} / \hbar$ with $\mathbf{d}_{e g}=\langle e|\mathbf{d}| g\rangle$. The dressed states are the eigenstates in the absence of spontaneous emission and for atomic velocity sufficiently small that nonadiabatic transitions between dressed states can be ignored. Neglecting spontaneous emission, the densitymatrix equations in the dressed-state basis for an atom moving with velocity $v$ along the $z$ axis are

$$
\begin{aligned}
& \frac{d \rho_{11}}{d t}=v \nabla \theta\left(\rho_{12}+\rho_{21}\right), \\
& \frac{d \rho_{12}}{d t}=-i \Omega \rho_{12}+v \nabla \theta\left(\rho_{22}-\rho_{11}\right),
\end{aligned}
$$

with

$$
\nabla \theta=-\frac{k \Delta \Omega_{0} \cos k z}{2 \Omega^{2}},
$$

where $\rho_{i j}=\langle i, n|\rho| j, n\rangle \quad(i, j=1,2), \quad \rho_{11}+\rho_{22}=1, \quad$ and $\rho_{21}=\rho_{12}^{*}$. The force on the atom is given by [7]

$f(z, v)=-\langle\nabla V\rangle=\frac{\hbar \nabla \Omega}{2}\left(\rho_{22}-\rho_{11}\right)-\hbar \Omega \nabla \theta\left(\rho_{12}+\rho_{21}\right)$,

with

$$
\nabla \Omega=\frac{k \Omega_{0}^{2} \sin 2 k z}{2 \Omega} .
$$

In the dressed-state picture, momentum diffusion due to stimulated processes is related to the fluctuation of the dipole force, which originates from spontaneous emission $[7,16]$. An atom in one dressed state can spontaneously decay to another dressed state, causing the force on the atom to change discontinuously in time. In between two spontaneous-emission events, the force on the atom changes continuously in time, and there is no force fluctuation. The accumulated effect of the fluctuating force on the atomic motion will lead to momentum diffusion. However, from a microscopic perspective each stimulated or spontaneous transition leads to momentum recoil, which can contribute to momentum diffusion. Therefore, treating diffusion in the dressed-state picture is an approximation, which is only valid for time scales much larger than $\tau$. This average over the quantum unit $\hbar k$ of momentum exchange between the atom and field is consistent with the treatment of diffusion given in Refs. [6] and [7]. The momentum diffusion due to spontaneous emission is much smaller than that due to the fluctuations of the dipole force for a high-intensity field and are ignored in this paper.

A Monte Carlo method can be used to simulate momentum diffusion in the dressed-atom picture. Consider an atom in a dressed state initially moving with velocity $v$. In between two spontaneous emissions, the density-matrix elements are given by the time integration of Eq. (4), and the atomic motion is determined by the instantaneous force given by Eq. (5). Since the dressed states are not exact eigenstates for a moving atom, the atom will be in a superposition of both dressed states. At each time step $t \rightarrow t+\Delta t$, the spontaneous decay probability from the superposition state to each dressed state is calculated as the product of the decay rate and $\Delta t$. If the superposition is given by $|\psi\rangle=c_{1}|1, n\rangle+c_{2}|2, n\rangle$, then the corresponding decay rates $\gamma_{1}$ and $\gamma_{2}$ from $|\psi\rangle$ to the lower manifold dressed states $|1, n-1\rangle$ and $|2, n-1\rangle$ are determined by the matrix elements of the atomic dipole moment $\langle 1, n-1|d| \psi\rangle=i d_{g e} \sin \theta\left(c_{1} \cos \theta-c_{2} \sin \theta\right)$ and $\langle 2, n-1|d| \psi\rangle=i d_{g e} \cos \theta\left(c_{1} \cos \theta-c_{2} \sin \theta\right)$ using Eq. (3):

$$
\begin{aligned}
\gamma_{1} & =\gamma \sin ^{2} \theta\left|c_{1} \cos \theta-c_{2} \sin \theta\right|^{2} \\
& =\gamma \sin ^{2} \theta\left[\rho_{11} \cos ^{2} \theta-\left(\rho_{12}+\rho_{21}\right) \cos \theta \sin \theta+\rho_{22} \sin ^{2} \theta\right],
\end{aligned}
$$

$\gamma_{2}=\gamma \cos ^{2} \theta\left[\rho_{11} \cos ^{2} \theta-\left(\rho_{12}+\rho_{21}\right) \cos \theta \sin \theta+\rho_{22} \sin ^{2} \theta\right]$.

Two pseudorandom numbers are generated by a computer to determine if a decay to state 1 or 2 happens during the time $\Delta t$. If a decay occurs, the initial conditions are reset according to the state the atom decays to. The momentum of the atom can be thus obtained for any interaction time. After many independent simulations, corresponding to many atoms, the desired momentum distribution is produced, with some statistical uncertainty.

As a check, the Monte Carlo method can be used to calculate the time-averaged force on an atom which can be compared with that obtained by the numerically exact continued-fraction method [4,5]. This comparison is made in Fig. 2 for $\Omega_{0}=50 \gamma$ and $\Delta=+15 \gamma$. For the Monte Carlo calculation, the instantaneous force on an atom moving with a fixed momentum is averaged over many wavelengths and many spontaneous emissions. These two calculations agree reasonably well for $|p| \leq p_{c}$. This Monte Carlo method is similar to a previously published method [20], except that in the expression for

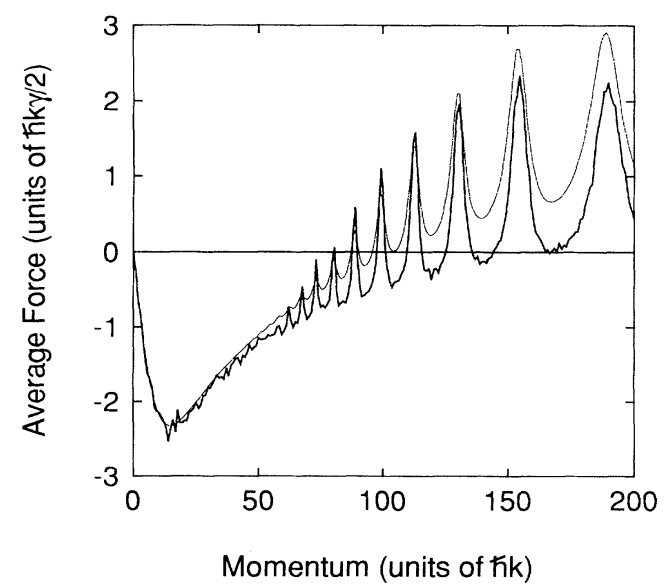

FIG. 2. Comparison of the average force on an atom in a standing wave vs the atom's momentum using the Monte Carlo method (bold curve) and the continued-fraction method (lighter curve). The Rabi frequency is $\Omega_{0}=50 \gamma$, and the detuning is $\Delta=+15 \gamma$. The two calculations agree reasonably well up to the critical momentum $p_{c}$. 
$d \rho_{12} / d t$ in Eq. (4) we include a velocity-dependent term that allows for the development of coherence between the dressed states due to the atomic velocity. This added term increases the velocity range over which the calculation is applicable since it accounts for the velocityresonant, nonadiabatic transitions, which give rise to the dopplerons [23]. In particular, the fact that the force changes sign at $p_{c}$ is correctly modeled.

The discrepancy between the two calculations shown in Fig. 2 for large $p$ is systematic. The only effect of spontaneous emission in our Monte Carlo simulations is to project the atom onto dressed state 1 or 2 . However, spontaneous emission will damp the excited-state population between spontaneous emission events [22], so that our treatment is exact only when the dressed states are exact eigenstates of the Hamiltonian that includes the interaction of the atom with the vacuum field. The dressed-state Monte Carlo method is best for slowly moving atoms in an intense, near-resonant standing wave, since the stimulated transition rates are high and the dressed states are a good approximation to the true eigenstates. At momenta corresponding to doppleron resonances, the rate for stimulated multiphoton Raman transitions can be much higher than $\gamma$, so that there is relatively better agreement at these momenta and poorer agreement at large $p$ between doppleron resonances.

\section{EXPERIMENT}

\section{A. Experimental setup}

A schematic of the experimental setup is shown in Fig. 3. An oven heated to $\sim 570^{\circ} \mathrm{C}$ produces a beam of $\mathrm{Li}$ atoms directed along the $x$ axis. The atomic beam is intersected at $90^{\circ}$ by a standing wave (along the $z$ axis) which is formed by retroreflecting the output from a fre-

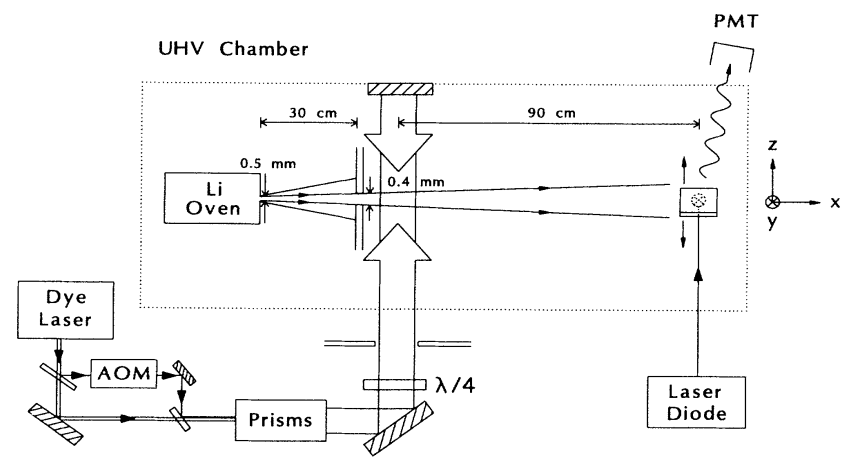

FIG. 3. Schematic diagram of the apparatus. A thermal beam of $\mathrm{Li}$ atoms (along the $x$ axis) is intersected at $90^{\circ}$ by an intense standing wave (along the $z$ axis), whose frequency is tuned near the $2 s_{1 / 2}, F=2 \leftrightarrow 2 p_{3 / 2}, F=3$ resonance frequency of the ${ }^{7} \mathrm{Li}$ atom (671-nm wavelength). A small fraction of the laser beam is shifted with an acousto-optic modulator (AOM) to optically pump atoms out of $F=1$ ground state. The laser beam is expanded along the $x$ axis by six prisms and circularly polarized by a quarter-wave plate $(\lambda / 4)$. The transverse momenta of the atoms are probed downstream of the interaction region using a weak, focused beam from a diode laser. Fluorescence from the atoms is detected by a photomultiplier tube (PMT). quency stabilized cw ring dye laser. The laser is tuned near the $2 s_{1 / 2}, F=2 \leftrightarrow 2 p_{3 / 2}, F=3$ resonance frequency of the ${ }^{7} \mathrm{Li}$ atom (671 $\mathrm{nm}$ wavelength). An effective two-state cycling system is realized using a quarter-wave plate to circularly polarize the standing wave so that atoms in the $F=2, m_{F}=2$ ground state can only be excited to the $F=3, m_{F}=3$ excited state. Atoms not initially in the $F=2, m_{F}=2$ ground state will be optically pumped either into this state from which they can participate in the two-state cycling process, or into the $F=1$ ground-state hyperfine level. The laser beam is collimated by a telescope, and expanded along the $x$ axis by six prisms to increase the interaction time with the atoms. The $x$-axis Gaussian beam waist ( $e^{-2}$ point of the maximum intensity) is $11.2 \mathrm{~mm}$ while the $y$-axis beam waist is $0.73 \mathrm{~mm}$. The laser output power is $510 \mathrm{~mW}$, giving a peak intensity in the standing wave of $16 \mathrm{~W} / \mathrm{cm}^{2}$. The saturation intensity (i.e., $\Omega_{0}=\gamma$ ) of the $F=2, m_{F}=2 \leftrightarrow F=3, m_{F}=3$ transition using circularly polarized light is $5.1 \mathrm{~mW} / \mathrm{cm}^{2}$, yielding a peak on-resonance Rabi frequency $\Omega_{0}=56 \gamma$. The central part of laser beam can be apertured along the $x$ axis to different lengths corresponding to different interaction times as atoms pass through the laser beam. The maximum variation of $\Omega_{0}$ over the unapertured part of the beam is $25 \%$. The laser frequency is offset-locked using saturated absorption spectroscopy in a heat pipe from the $2 s_{1 / 2}, F=2 \leftrightarrow 2 p_{3 / 2}, F=3$ resonance frequency of the ${ }^{7} \mathrm{Li}$ atom, so that the detuning is accurately determined. A small fraction of the main beam is frequency shifted with an acousto-optic modulator (AOM), in order to pump atoms out of the $F=1$ state (ground-state hyperfine splitting of $803 \mathrm{MHz}$ ). This AOM beam is aligned nearly collinear to the main beam. The AOM beam power is $15 \mathrm{~mW}$ with a beam waist approximately equal to that of the main beam. The interaction of the atoms with the standing wave defines the quantization axis, since this interaction is stronger than that due to any other field, including the Earth's magnetic field. To further eliminate the background magnetic field, a high- $\mu$ metal tube is placed around the interaction region, collinear with the standing-wave axis.

The effect of the standing wave on the transverse velocities of the atoms in the beam is probed downstream of the interaction region using a weak, focused beam from a diode laser. The probe beam is parallel to the $x-y$ plane and intersects the atomic beam nearly orthogonally, at an angle of $\sim 87^{\circ}$ relative to the atomic beam axis. The probe laser frequency is offset-locked from the $2 s_{1 / 2}, F=2 \leftrightarrow 2 p_{3 / 2}, F=3$ resonance frequency by 110 $\mathrm{MHz}$, so the probe will preferentially excite atoms with a longitudinal velocity centered around $1400 \mathrm{~m} / \mathrm{s}$, with a Lorentzian width of $75 \mathrm{~m} / \mathrm{s}$ due to the linewidth of the transition. The atomic fluorescence induced by the probe beam is collected by a lens, detected by a photomultiplier and individual photons are counted and recorded by a computer. The position of the probe beam is scanned in the $z$ direction, across the atomic beam, using a mirror mounted on a translation stage to record the transverse distribution of the atoms in the beam [24]. For a single longitudinal velocity, there is a one to one correspondence between transverse position and transverse veloci- 
ty. The Gaussian beam waist of the focused probe beam of $0.2 \mathrm{~mm}$, together with the dimension of the collimating slit yield a transverse velocity resolution of $0.6 \mathrm{~m} / \mathrm{s}$, or equivalently, a momentum resolution of $7 \hbar k$ $(\hbar k / M=8.5 \mathrm{~cm} / \mathrm{s})$.

\section{B. Data with Monte Carlo simulations}

Figures 4-6, 8, 9(a), and 12(a) show experimentally measured momentum distributions (bold lines) and the results of Monte Carlo simulations (lighter lines) for various interaction times $t$, detunings $\Delta$, and for a spatial average along the $x$ axis of $\Omega_{0}$ over the unapertured part of the standing wave of $\left\langle\Omega_{0}\right\rangle \approx 50 \gamma$. The Monte Carlo simulations have no adjustable parameters other than the center point $(p=0)$ of the distribution and the normalization. The simulation results shown in Figs. 4, 5, 8, 9(a), and 12 (a) correspond to trajectories of $\sim 10^{4}$ atoms, while 2000 atoms are used for the simulation results in Figs. 6 and 7.

Figure 4 shows results for $\Delta=+5 \gamma$, and for interaction times of (a) $t=0$ and (b) $t=320 \tau$. The vertical axis of these plots represents the probe-induced fluorescence, which is proportional to the number of atoms. The horizontal axis is the transverse position of the probe beam,

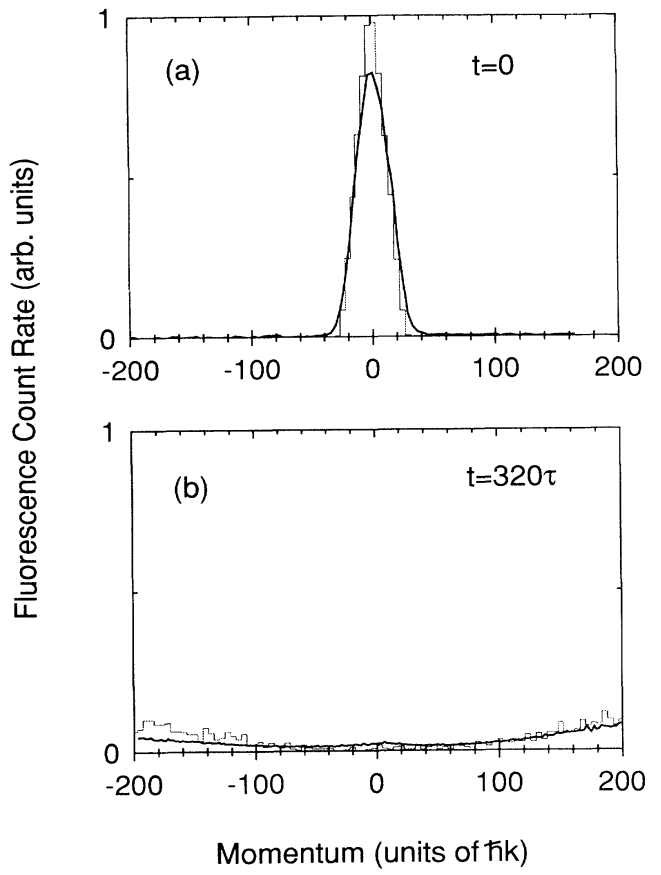

FIG. 4. Data (bold curves) and results of the Monte Carlo simulation (lighter lines) for the transverse momentum distribution of atoms in the beam. The vertical axis is the fluorescence induced by the weak probe laser, which is Doppler tuned to a single longitudinal velocity $(\sim 1400 \mathrm{~m} / \mathrm{s})$. The horizontal axis is the transverse position of the probe beam which is scaled to the transverse momentum in units of $\hbar k(\hbar k / M=8.5 \mathrm{~cm} / \mathrm{s})$. The standing-wave parameters are $\left\langle\Omega_{0}\right\rangle \approx 50 \gamma$ and $\Delta=+5 \gamma$. The interaction times are (a) $t=0$ and (b) $t=320 \tau$. The data show that because of large diffusion, most of the atoms escape the confined region $|p|<p_{c}$ defined by the dipole force by $t=320 \tau$. and is scaled to the transverse momentum in units of $\hbar k$. The stimulated transition rate for one traveling-wave component of the standing wave for an atom with velocity $\mathbf{v}$ is $R=\frac{1}{4} \gamma \Omega_{0}^{2} /\left[4(\Delta-\mathbf{k} \cdot \mathbf{v})^{2}+\gamma^{2}\right]$. As discussed earlier, momentum diffusion is predominantly caused by stimulated transitions randomly involving each traveling wave. Therefore, the rate of diffusion will depend on $R$ of both traveling waves. As $R$ increases, the number of stimulated transitions increases, thereby increasing the rate of momentum diffusion. Since an atom with $\mathbf{v} \neq 0$ sees the two traveling-wave components Doppler shifted by unequal amounts, $\pm|\mathbf{k} \cdot \mathbf{v}|$ respectively, $R$ for the component which is Doppler shifted further from resonance is reduced compared to $R$ for $\mathbf{v}=0$. Therefore, the rate of momentum diffusion is largest at $\mathbf{v}=0$, and decreases for larger $\mathbf{v}$. For the conditions of Fig. $4, R$ is large because $\Delta$ is relatively small, and momentum diffusion is seen to dominate. Although the dipole force is cooling for $|p|<p_{c}(\sim 25 \hbar k)$, those atoms rapidly diffuse to $|p|>p_{c}$ where the force changes sign, and are then further heated. Therefore, there is no steady-state momentum distribution.

Figure 5 corresponds to $\Delta=+9 \gamma$, and (a) $t=0$, (b) $t=40 \tau$, and (c) $t=320 \tau$. As $\Delta$ increases from $5 \gamma$ to $9 \gamma$, $R$ is reduced, so that the rate of the momentum diffusion decreases. However, the maximum dipole force changes little, while $p_{c}$ is nearly doubled. Consequently, the force is able to slow the rate of diffusive loss at larger momenta, where the rate of momentum diffusion is smaller.

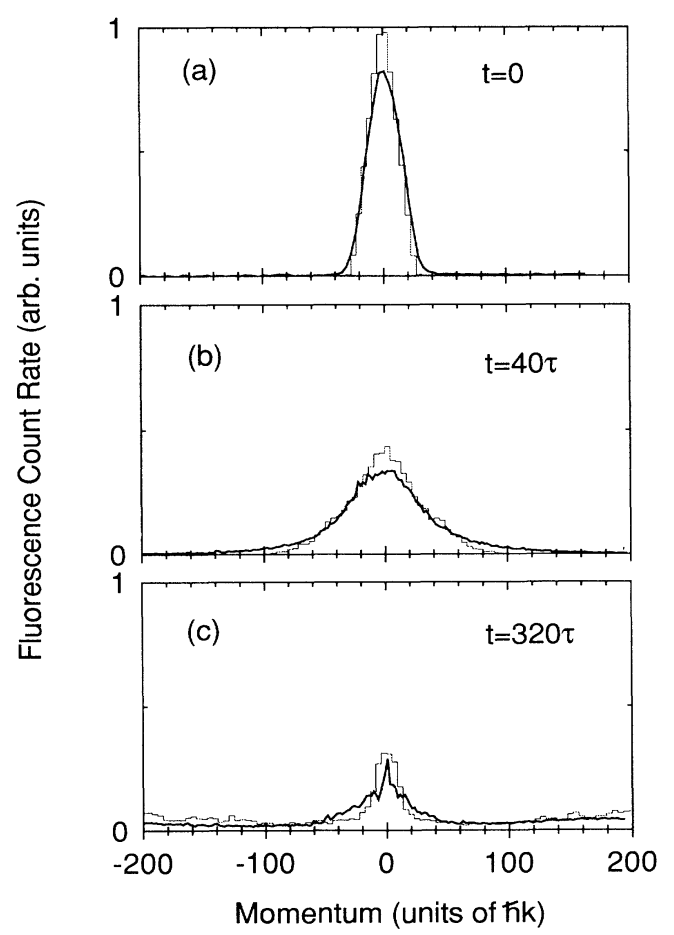

FIG. 5. Data (bold curves) and simulations (lighter curves) for $\Omega_{0}=50 \gamma, \Delta=+9 \gamma$, and for (a) $t=0$, (b) $t=40 \tau$, and (c) $t=320 \tau$. For this detuning, the rate of loss of atoms from the confined region is smaller than that of Fig. 4 , but still there is no steady-state distribution. 
Again, there is no steady state, though the rate for diffusion to higher momentum is reduced.

Figure 6 corresponds to $\Delta=+15 \gamma$, and (a) $t=0$, (b) $t=40 \tau$, and (c) $t=320 \tau$. Diffusion is further diminished in comparison to the $\Delta=5 \gamma$ and $9 \gamma$ cases, and can be effectively balanced by the force. Note that for blue detuning there should be no exact steady state because atoms will eventually diffuse to $|p|>p_{c}$, where they experience a time-averaged heating force. However, the rate of diffusion is sufficiently slow that a quasiequilibrium is established for time scales of experimental interest. Figure 7 shows Monte Carlo simulation results for $\Delta=+15 \gamma$, and for (a) $t=0$, (b) $t=320 \tau$, (c) $t=1000 \tau$, (d) $t=2000 \tau$, and (e) $t=5000 \tau$. For the distribution at $t=320 \tau$, all the atoms are confined to the region of $|p|<p_{c}(\sim 80 \hbar k)$, and the distribution has a root-meansquared (rms) momentum of $22 \hbar k$, which corresponds to a temperature of $3 \mathrm{mK}$ (the Doppler cooling limit is 0.14 $\mathrm{mK})$. The distribution becomes noticeably narrower between $t=320 \tau$ and $2000 \tau$, at which point $99.7 \%$ of atoms remain with $|p|<p_{c}$, and the distribution has a rms momentum of $18 \hbar k$. It will be shown in Sec. IV B that the rate of cooling which produces the noticeably narrow feature for $t \geq 2000 \tau$ is determined by the time scale for localizing, or "channeling" atoms around the standingwave nodes. The two small peaks around $\pm 6 \hbar k$, which become more pronounced for $t \geq 2000 \tau$, are an artifact of this semiclassical calculation and are discussed in Sec. IV B.

Figure 8 corresponds to $\Delta=-10 \gamma$ (i.e., red detuning), and (a) $t=0$, (b) $t=20 \tau$, (c) $t=40 \tau$, (d) $t=80 \tau$, (e)

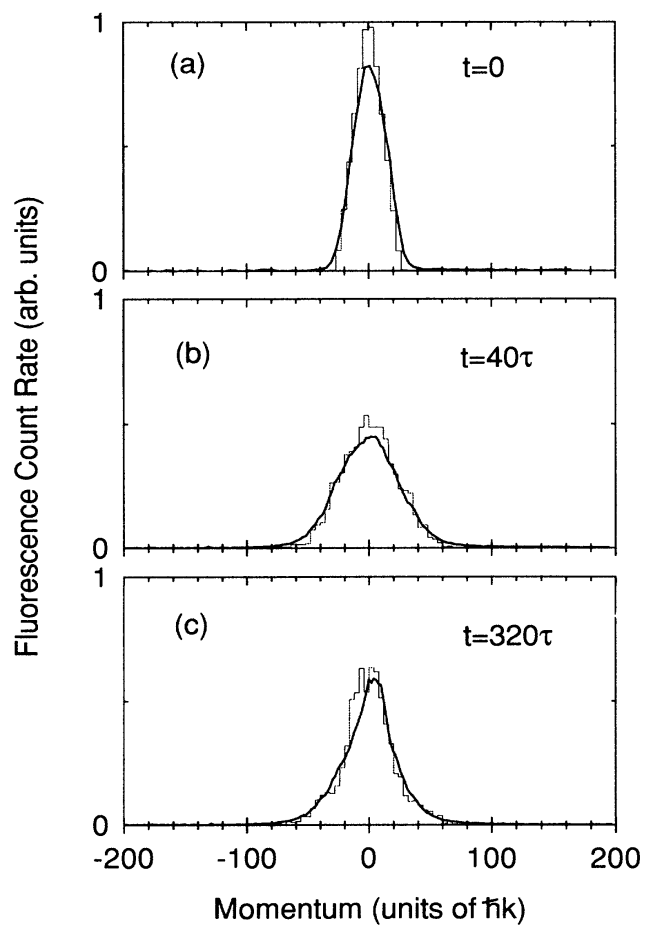

FIG. 6. Data (bold curves) and simulations (lighter curves) for $\Omega_{0}=50 \gamma, \Delta=+15 \gamma$, and for (a) $t=0$, (b) $t=40 \tau$, and (c) $t=320 \tau$. In this case, there is virtually no loss of atoms from the confined region up to $t=320 \tau$. $t=160 \tau$, and (f) $t=320 \tau$. Here, the force is heating for $|p|<p_{c}(\sim 55 \hbar k)$, and cooling for $|p|>p_{c}$. Therefore, for red detuning, the time-averaged force will balance the diffusive heating, resulting in a true steady state. The steady state is reached by $t=160 \tau$. If there were no diffusion, atoms would be bunched in two narrow spikes at $p= \pm p_{c}$. Thus, the widths of the two peaks centered near $p= \pm p_{c}$ are a consequence of diffusion. The Monte Carlo simulations exhibit the correct time evolution of the momentum distribution.

The data and simulation results for $\Delta=-2 \gamma$ and $t=320 \tau$ are shown in Fig. 9(a). The initial distribution is the same as for the previous data. For this case, $p_{c}$ occurs at a relatively small value, $\sim 10 \hbar k$, where the diffusion is large. Therefore, no resolvable peaks at $p= \pm p_{c}$ are expected. Again, the steady state is reached by $t=320 \tau$. However, the small central dip appearing in the data, which is not seen in the simulation, may be due to the relatively large uncertainty in $\Delta$; if the detuning were slightly redder than believed, the critical momenta would be
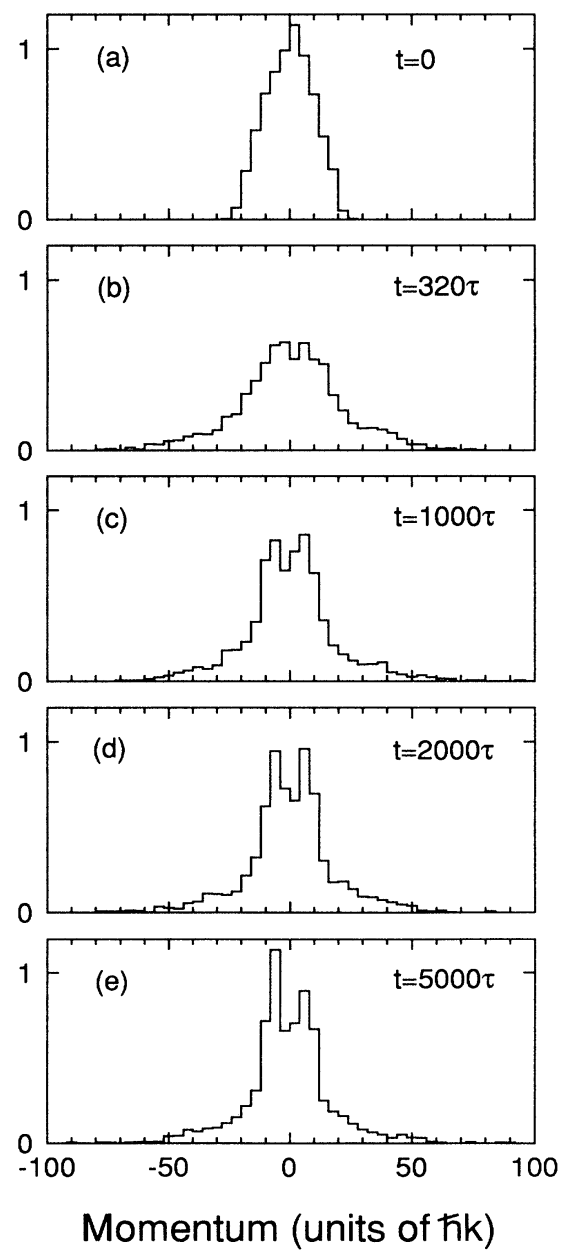

FIG. 7. Monte Carlo simulations for $\Omega_{0}=50 \gamma$ and $\Delta=+15 \gamma$, and for (a) $t=0$, (b) $t=320 \tau$, (c) $t=1000 \tau$, (d) $t=2000 \tau$, and (e) $t=5000 \tau$. The long-term evolution shows narrowing of the atomic distribution due to the dipole force cooling and channeling of atoms. A quasiequilibrium is established for $t \sim 2000 \tau$. 
further separated, enabling the two peaks to be resolved. Discussion of Fig. 9(b) will be given in Sec. IV C.

It should be noted that a $\mathrm{Li}$ atom has a relatively small mass, so that velocity changes due to either the force or diffusion are large compared with that of larger mass atoms. Therefore, we are able to observe situations in which the diffusion completely dominates (e.g., Fig. 4), as well as steady-state distributions [e.g., Figs. 8 and 9(a)] using interaction times of only a few hundred $\tau$.

The data for the various experimental conditions generally agree with the Monte Carlo simulations quite well. However, as discussed in Sec. II, the dressed-state Monte Carlo method begins to fail for large $\Delta$, and for large $p$ between doppleron resonances. In these regions, the stimulated excitation rate is very small. There are slight discrepancies between the data and simulations that are

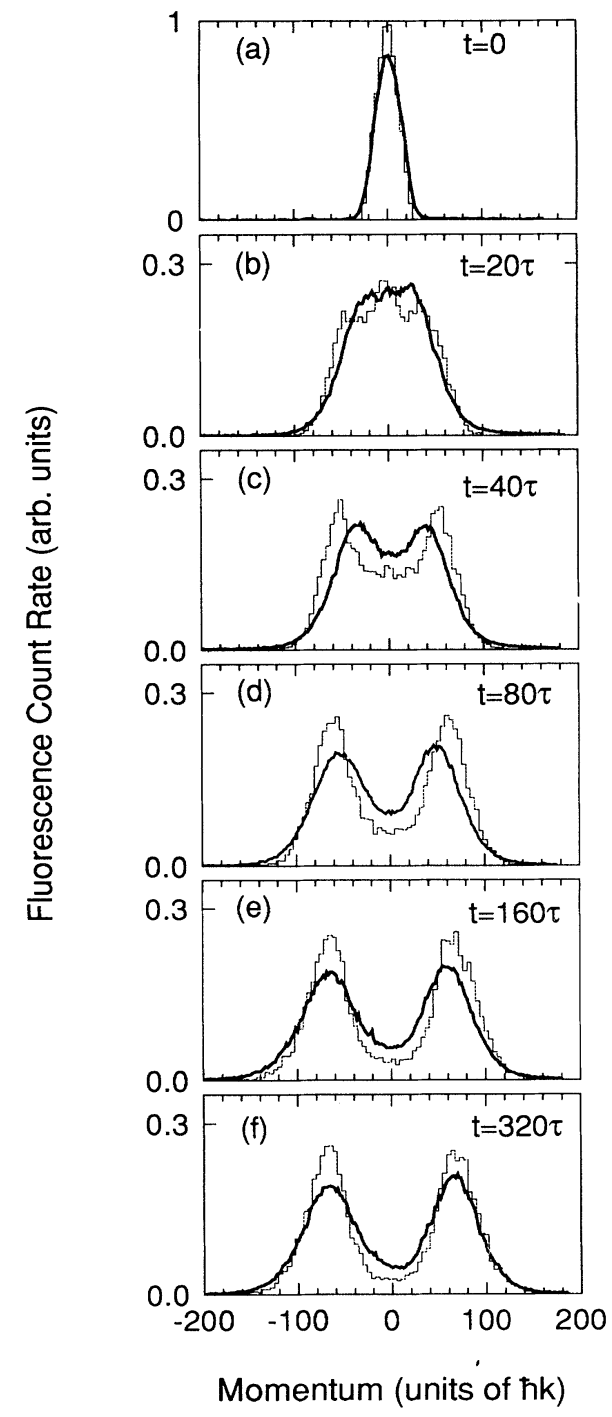

FIG. 8. Data (bold curves) and simulations (lighter curves) for $\Omega_{0}=50 \gamma, \Delta=-10 \gamma$, and for (a) $t=0$, (b) $t=20 \tau$, (c) $t=40 \tau$, (d) $t=80 \tau$, (e) $t=160 \tau$, and (f) $t=320 \tau$. The true steady-state distribution is reached by $t=160 \tau$. Two peaks are centered near $\pm p_{c}$ where the atoms would be bunched if there were no diffusion.
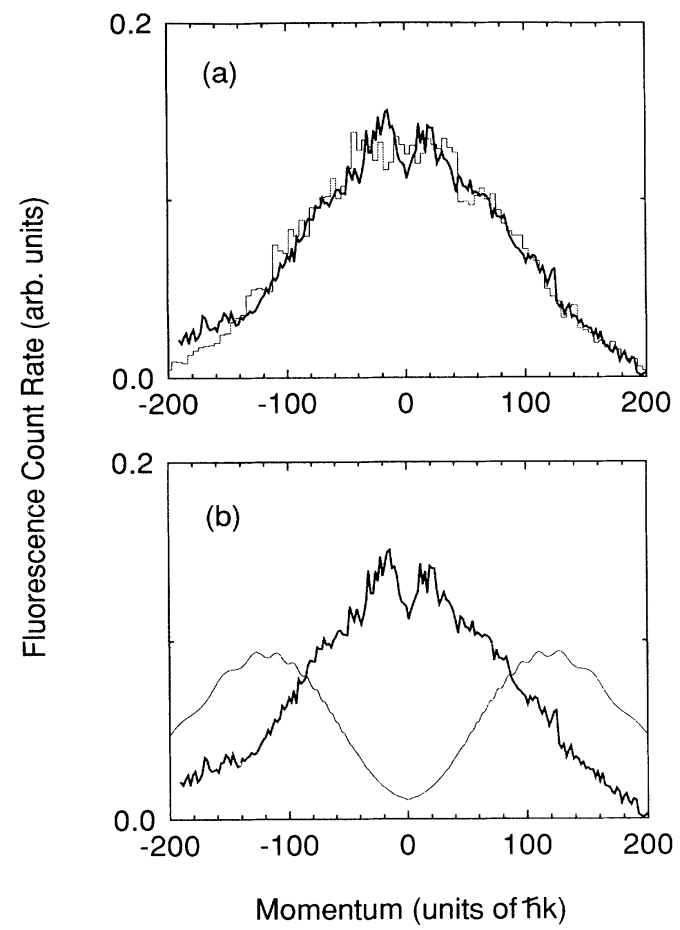

FIG. 9. $\Omega_{0}=50 \gamma$ and $\Delta=-2 \gamma$. (a) Data (bold curve) and Monte Carlo simulation (lighter curve) for $t=320 \tau$. (b) Data (bold curve) at $t=320 \tau$ and the steady-state solution of the Fokker-Planck equation (lighter curve). For this small detuning, the Fokker-Planck equation obviously fails to reproduce the data.

most pronounced for $\Delta=-10 \gamma$. These discrepancies may be indicative of the reduced validity of the Monte Carlo method.

Another effect that needs to be accounted for is the reduction of the effective interaction times with the standing wave due to optical pumping. The ground-state populations are initially distributed among the ground state hyperfine levels $F=1$ and 2 with a statistical weighting factor of $2 F+1$. It take some time to optically pump all the atoms into the $F=2, m_{F}=2$ state. Once atoms are in the $F=2, m_{F}=2$ state, they participate in the two-state cycling transition $F=2, m_{F}=2 \leftrightarrow F$ $=3, m_{F}=3$. This optical pumping time will reduce the interaction time with the standing wave. We determined the average reduction in interaction time for a given $\Omega_{0}$ and $\Delta$ by evaluating the integral over the entire momentum distribution of the probe-induced fluorescence for different interaction times with the standing wave. The quoted interaction times are the actual times for atoms to pass through the standing wave, but the interaction times for the simulations are reduced to account for this effect. The optical pumping times range between $t=0$ and $20 \tau$ for our data, depending on $\Omega_{0}$ and $\Delta$. Slight discrepancies between the data and simulations for small $t$ may be attributed to uncertainties in this effect. 


\section{DISCUSSION}

\section{A. Broadening due to the standing-wave potentials}

Interestingly, the distribution in Fig. 6 first becomes broader at $t=40 \tau$, and then narrower at $t=320 \tau$. As the ground-state atoms initially enter the standing wave, they will experience a sudden rise in their internal potential energy whose magnitude depends on whether they evolve into either dressed state $|1, n\rangle$ or $|2, n\rangle$ [the probability for each can be derived from Eq. (3)]. The potentialenergy functions $U_{1}(z)$ and $U_{2}(z)$, corresponding to the $|1, n\rangle$ and $|2, n\rangle$ states, respectively, can be derived from the total energies $E_{1 n}$ and $E_{2 n}$ [Eq. (2)]:

$$
\begin{aligned}
& U_{1}(z)=\frac{\hbar}{2}\left[\left(\Omega_{0}^{2} \sin ^{2} k z+\Delta^{2}\right)^{1 / 2}-|\Delta|\right], \\
& U_{2}(z)=\frac{\hbar}{2}\left[\Omega_{m}-\left(\Omega_{0}^{2} \sin ^{2} k z+\Delta^{2}\right)^{1 / 2}\right],
\end{aligned}
$$

where $\Omega_{m}=\left(\Omega_{0}^{2}+\Delta^{2}\right)^{1 / 2}$. The depth of the potential wells is $U_{m}=\frac{1}{2} \hbar\left(\Omega_{m}-|\Delta|\right)$. $U_{1}$ and $U_{2}$ are scaled so that $U_{1}, U_{2} \geq 0$.

The atomic potential energy can be raised significantly, especially for atoms in the $|1, n\rangle$ state near antinodes [where $U_{1}(z)$ reaches its maximum] and for atoms in the $|2, n\rangle$ state around nodes [where $U_{2}(z)$ is at its maximum]. As the atoms move, their potential energy is converted to kinetic energy, resulting in a broadening of the momentum distribution. The broadening occurs on the time scale for an atom to move one optical wavelength. For the momentum range of interest, this will occur within several $\tau$. The rms momentum $\left\langle\Delta p^{2}\right\rangle^{1 / 2}$ increases by $\sim 20 \hbar k$ due to this effect alone. However, the distribution is not significantly affected by the dissipative dipole force or diffusive heating for $t<40 \tau$. In classical diffusion theory, the change of kinetic energy of an atom due to the time-averaged force and the time-averaged diffusion is determined by $d\left(p^{2} / 2 M\right) / d t=F^{0}(v) v$ $+D^{0}(v) / M[6]$, where $F^{0}(v)$ and $D^{0}(v)$ are the spatially averaged velocity-dependent force and the velocitydependent momentum diffusion coefficient, respectively [5]. A numerical integration of the above equation for $\Omega_{0}=50 \gamma$ and $\Delta=15 \gamma$, and for atoms with $|p|<p_{c}$, shows a time scale of $200 \tau$ for the dissipative force to cool the atoms to the quasiequilibrium distribution determined by the balance of the dissipation and diffusion. Broadened distributions at $t=40 \tau$ for both $\Delta=+15 \gamma$ and $+9 \gamma$ are attributed to this sudden rise in potential energy. By $t=320 \tau$, the atoms have been cooled substantially by the dissipative dipole force, narrowing the distribution.

\section{B. The dynamics of atom channeling}

For $\Delta>0$, an atom in the $|1, n\rangle$ state slowly moving in a standing wave can be confined to, or channeled in, the nodes of the standing wave, while for $\Delta<0$, an atom in the $|2, n\rangle$ state can be channeled around the antinodes if, in either case, the maximum kinetic energy of the atom $\left(p_{m}^{2} / 2 M\right)$ is less than the potential depth $U_{m}[18-20,25]$.

Figure 10 shows the result of a dressed-state Monte

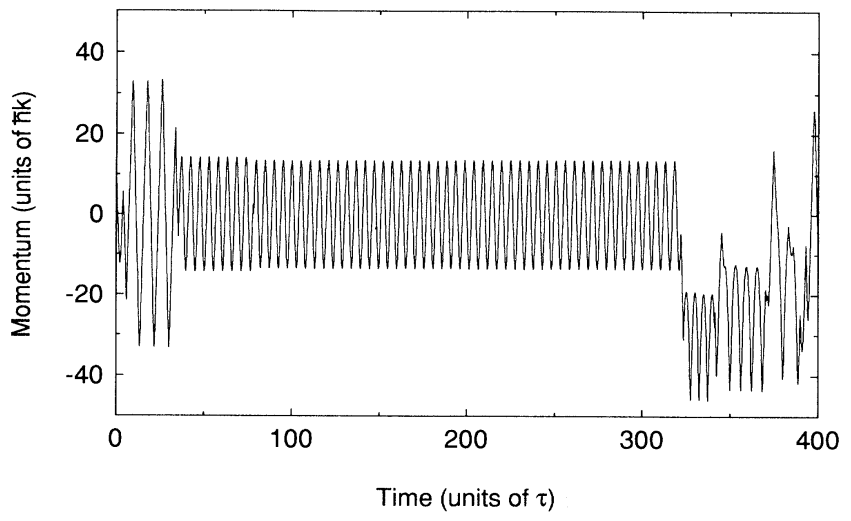

FIG. 10. Momentum vs time for the Monte Carlo simulation of a single atom for $\Omega_{0}=50 \gamma$ and $\Delta=+15 \gamma$. The atomic motion is periodic with interruptions caused by spontaneous emission events. The periodic oscillation around zero is clear evidence of channeling. An occasional spontaneous emission can cause the atom to become unchanneled.

Carlo simulation of the momentum of a single atom versus time for $\Omega_{0}=50 \gamma$ and $\Delta=15 \gamma$. For these parameters, $U_{m}=19 \hbar \gamma$, and the maximum channeled momentum $p_{m}=41 \hbar k$, which is determined by $p_{m}^{2} / 2 M=U_{m}$. In the dressed-state picture, the atomic motion is periodic, with interruptions caused by spontaneous-emission events. In this particular simulation, the atom spontaneously decays near $t=6 \tau$ and is left channeled in the $|1, n\rangle$ state with an oscillation amplitude of $33 \hbar k$. Later, the atom spontaneously decays at $t=34 \tau$. Then at $t=36 \tau$, and again at $t=80 \tau$, spontaneous emission leaves the atom more deeply channeled. Since the $|1, n\rangle$ state contains only a small component of the excited state at low field intensity, the more deeply an atom channels, the smaller the probability for spontaneous decay. In addition, when spontaneous emission occurs, the decay is most likely to the $|1, n-1\rangle$ state, in which the atom may remain channeled. The atom does not spontaneously emit during the interval $t=80 \tau$ to $320 \tau$. During this interval, it oscillates with maximum momentum of $13 \hbar k$ and with a period of $5 \tau$. The atom finally decays to the $|2, n-1\rangle$ state at $t=320 \tau$, causing it to become unchanneled.

The dressed-atom model is quite appropriate for describing the motion of a deeply channeled atom since the rate of spontaneous decay is very small compared to the atom's oscillation frequency. Therefore, between spontaneous-emission events the atom experiences the dressed-state potentials $U_{1}(z)$ and $U_{2}(z)$ [Eq. (7)]. In order to relate this work to previous investigations of atom channeling, we note that the potential obtained by averaging the dressed-state potentials over many spontaneous-emission events weighted by the relative probability of occupying each dressed-state results in an effective potential $U_{\text {eff }}(z)=\frac{1}{2} \hbar \Delta \ln \left[1+2 \Omega_{0}^{2} \sin ^{2} k z /\left(4 \Delta^{2}\right.\right.$ $\left.+\gamma^{2}\right)$ ] [26]. This potential is appropriate for an atom in a standing wave when the spontaneous-emission rate is sufficiently high that the atom does not move a significant fraction of a wavelength between spontaneous-emission 
events.

In order to describe the channeling quantitatively, the mean lifetime of a channeled atom for the blue detuning case $(\Delta>0)$ can be evaluated. Let $\gamma_{2}$ be the spontaneous-emission rate from the $|1, n\rangle$ state to the $|2, n-1\rangle$ state. Since $\gamma_{2}$ represents decay to the unconfined state, the time average of $\gamma_{2}$ over an oscillation period yields the mean lifetime $T_{\mathrm{ch}}$ :

$$
T_{\mathrm{ch}}=\left\langle\gamma_{2}\right\rangle^{-1}=\left\langle\cos ^{4} \theta\right\rangle^{-1} \tau
$$

where the expression for $\gamma_{2}$ is obtained from Eq. (6) and $\theta \equiv \theta(z(t))$ is determined by the motion of the atom in the potential $U_{1}(z)$. For example, for the atom in Fig. 10 , with maximum momentum of $13 \hbar k, T_{\mathrm{ch}}=215 \tau$.

The analysis given above treated the atomic motion classically. When an atom channels very deeply in the potential $U_{1}(z)$, its momentum is on the order of $\hbar k$ and its motion can no longer be considered classical. The quantized motion of atoms in optical potential wells, created by one-dimensional, polarization-gradient cooling, was recently observed [27]. If the oscillation amplitude $z_{0}<<\lambda / 2 \pi \min \left\{1, \Delta / \Omega_{0}\right\}$, the potential energy $U_{1}(z)$ can be approximated as a harmonic potential $U_{h}(z)$,

$$
U_{h}(z)=\frac{\hbar k^{2} \Omega_{0}^{2}}{4 \Delta} z^{2}=\frac{1}{2} M \omega^{2} z^{2},
$$

where $\omega=\Omega_{0} k[\hbar /(2 \Delta M)]^{1 / 2}$ is the harmonic oscillation frequency. For $\Omega_{0}=50 \gamma$ and $\Delta=15 \gamma, z_{0}=\lambda / 50$ and $\omega=1.3 \gamma$. The quantum-mechanical ground state of $U_{h}(z)$ has an energy $E_{0}=\frac{1}{2} \hbar \omega$ and a rms momentum $\left\langle\Delta p^{2}\right\rangle^{1 / 2}=5.7 \hbar k . \quad T_{\text {ch }}$ can be calculated quantum mechanically using Eq. (8) and the harmonic-oscillator ground-state wave function, giving $T_{\mathrm{ch}}=1120 \tau$.

The evolution of channeling was investigated by analyzing the ensemble of simulated atomic trajectories used to generate Fig. 7. In order to be quantummechanically consistent, the total energy $E$ of each atom in these semiclassical simulations is constrained to be $E \geq E_{0}$. An atom can be identified as channeled if it is in dressed state $|1, n\rangle$ and has total energy $E<U_{m}$. Whenever an atom channels more deeply than quantummechanically allowed $\left(E<E_{0}\right)$, its energy is manually raised to $E=E_{0}$. This ad hoc procedure produces the two artificial peaks in the momentum distribution shown in Fig. 7. The two peaks around $\pm 6 \hbar k$ correspond to the rms momentum of an atom channeled in the $n=0$ level. The fraction of channeled atoms (squares) as a function of $t$ is shown in Fig. 11. The dashed line is a fit to the calculational results using an exponential function with a time constant of $400 \tau$. Figure 11 also shows the fraction of atoms which are channeled in the " $n=0$ " (circles) and the " $n=1$ " (triangles) quantum-mechanical energy levels of $U_{h}(z)$, as a function of $t$. Atoms in the energy band $\frac{1}{2} \hbar \omega \leq E<\hbar \omega$ are identified with the $n=0$ level while those in the band $\hbar \omega \leq E<2 \hbar \omega$ are identified with the $n=1$ level. The continuous line is a fit to an exponential function with a time constant of $750 \tau$ for the $n=0$ level. It is evident that the narrowing of the distribution shown in Fig. 7 is a consequence of the increase in the number of

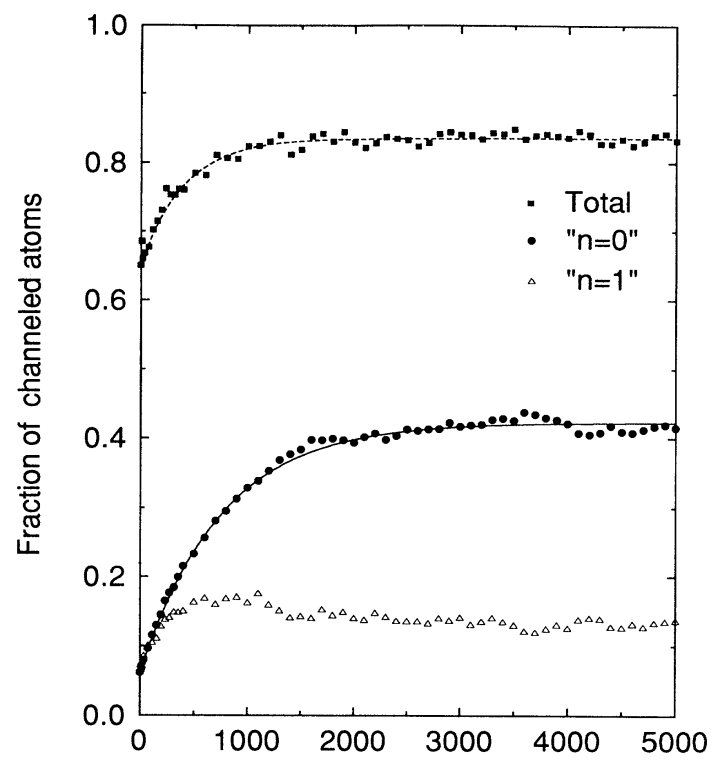

$t$ (units of $\tau$ )

FIG. 11. The ensemble of simulated atomic trajectories used to generate Fig. 7 is sorted to obtain the fraction of channeled atoms (squares), the fraction of the atoms channeled with an energy near to that of the $n=0$ quantum-mechanical energy level (circles), and the fraction channeled near the $n=1$ level (triangles), as a function of $t$. The dashed line is a fit to an exponential function with a time constant of $400 \tau$, while the continuous line is a fit to an exponential function with a time constant of $750 \tau$. A quasiequilibrium is established for $t \sim 2000 \tau$ in agreement with the results shown in Fig. 7.

channeled atoms, especially those in the $n=0$ level. A quasiequilibrium is reached when the rate to cool and load atoms into these channeled levels is balanced with the loss rate out of the channels due to spontaneous decay [Eq. (8)]. For the case of Fig. 11, the quasiequilibrium occurs at $t \sim 2000 \tau$, at which point the fraction of atoms channeled, the fraction in the $n=0$, and the fraction in the $n=1$ levels reach their constant values, $84 \%, 42 \%$ and $14 \%$, respectively. This time scale to reach the quasiequilibrium is consistent with the results shown in Fig. 7. Furthermore, the results shown in Figs. 7 and 11 are consistent with those of a similar calculation presented in Ref. [20], in which a narrow peak in the momentum distribution, attributed to channeled atoms, evolves with a time constant of several thousand $\tau$.

Understanding the dynamics of atom channeling is especially important for developing a new, recently demonstrated cooling method which is based on the adiabatic cooling of channeled atoms in an intense, bluedetuned standing wave [19]. It was demonstrated that channeled atoms can be cooled to a momentum near the single-photon recoil limit $\hbar k$ by adiabatic lowering the standing wave intensity. The Monte Carlo method was used to analyze this process. 


\section{The Fokker-Planck equation}

For long interaction times $t \gg \tau$ and large atomic momenta $p \gg \hbar k$, the Wigner density matrix equations can be used to represent the classical motion of an ensemble of atoms. The Wigner distribution can be shown to obey a Fokker-Planck equation (FPE) $[2,5,28]$. Various techniques have been developed to solve the FPE in order to calculate atomic momentum distributions [29]. One of the most effective ways is to transform the FPE into the Langevin equation and use Monte Carlo techniques to simulate the Langevin force. The transformed Langevin equation has the form [29]

$$
p(t+\Delta t)=p(t)+F^{0} \Delta t+r_{n}\left(D^{0} \Delta t\right)^{1 / 2},
$$

where $F^{0} \equiv F^{0}(p)$ and $D^{0} \equiv D^{0}(p)$ are the spatially averaged velocity-dependent force, and the velocitydependent momentum diffusion coefficient, respectively. $r_{n}$ is a Gaussian random variable with zero mean and variance 2 (i.e., $\left\langle r_{n}\right\rangle=0$ and $\left\langle r_{n}^{2}\right\rangle=2$ ). $F^{0}$ can be calculated by a continued-fraction method [4,5], and the numerical solution of $D^{0}$ can be obtained by integrating Eqs. (8.5) and (8.10) in Ref. [5] over many wavelengths, until all the transients die out, leaving the system in a periodic steady state. The second term on the right-hand side of Eq. (10) is the momentum change due to the deterministic force, while the last term describes the effect due to momentum diffusion, that is, the stochastic Langevin force. It becomes straightforward to obtain a momentum distribution for any initial distribution. For one simulation corresponding to a single atom, at every time interval $t \rightarrow t+\Delta t$, the atomic momentum change is given by Eq. (10). After many independent simulations, corresponding to many atoms, the desired momentum distribution is produced, with some statistical uncertainty.

The FPE approach is not applicable in the regime where $p \sim \hbar k$, since the atomic motion must be described quantum mechanically in this case. In addition, the spatially averaged $F^{0}$ and $D^{0}$ fail to account for the change in the atoms kinetic energy as they move between potential hill and valley. Therefore, this method should be most inaccurate for atoms with small $p$, for whose kinetic energy is comparable to $U_{m}$. In Fig. 9(b), the steady-state solution of the FPE [5] (lighter line) for $\Delta=-2 \gamma$ is compared with the data (bold line) for $t=320 \tau$. The discrepancy is large because a large fraction of the atoms have relatively small momenta. However, reasonably good agreement is found for large red detuning as shown in Fig. 12(b) for $\Delta=-10 \gamma$, since most of the atoms have relatively large momenta. Figure $8(\mathrm{f})$ is duplicated in Fig. 12(a) in order to compare the Monte Carlo result with the FPE calculation. The oscillations in the FPE result for $\Delta=-10 \gamma$ are due to the doppleron resonances. The Monte Carlo method and the Fokker-Planck equation should be complementary since they are best suited to opposite momentum regimes.

\section{CONCLUSION}

In summary, we have investigated the effect of the dipole force and its fluctuation (i.e., momentum diffusion) on the motion of $\mathrm{Li}$ atoms in an intense standing wave in
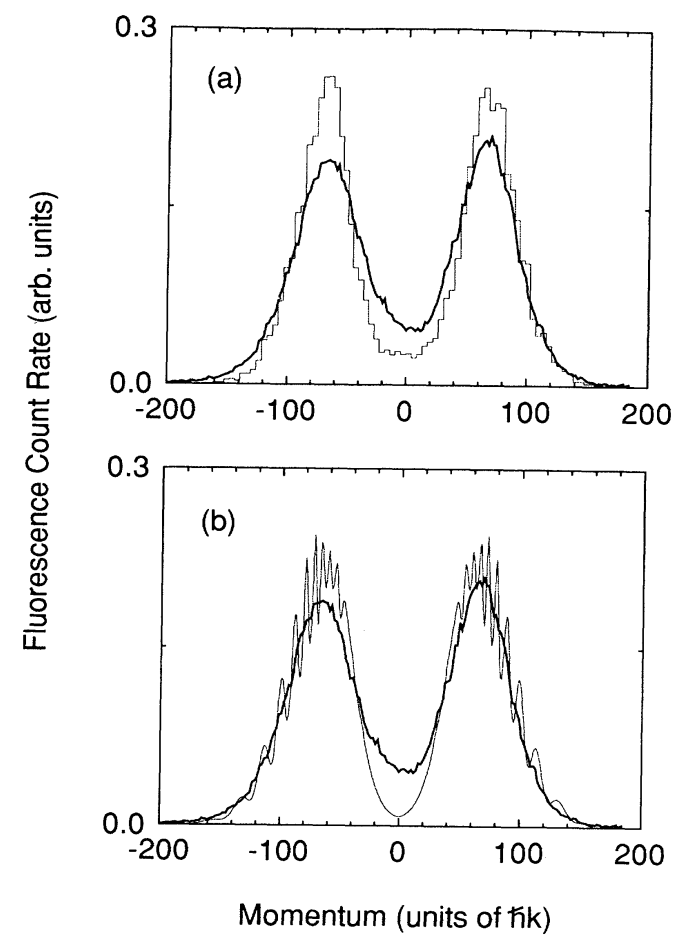

FIG. 12. $\Omega_{0}=50 \gamma$ and $\Delta=-10 \gamma$. (a) Data (bold line) and Monte Carlo simulation (lighter line) for $t=320 \tau$ [duplicate of Fig. 8(f)]. (b) Data (bold line) at $t=320 \tau$ and the steady-state solution of the Fokker-Planck equation (lighter line). For this detuning, both the Monte Carlo method and the Fokker-Planck equation agree with the data reasonably well.

the regime of high Rabi frequency $\Omega_{0} \gg \gamma$ and long interaction times $t \gg \tau$, and for atomic momenta $p \sim p_{c}$. We find that there is no exact steady-state momentum distribution for a blue-detuned standing-wave frequency. For small detunings $\left(\Delta<<\Omega_{0}\right)$, diffusive heating dominates the cooling provided by the time-averaged dipole force. However, for sufficiently large blue detunings, the rate of diffusion slows and a quasiequilibrium can be established on time scales of experimental interest. For red detunings, the diffusion is balanced by the force and a true steady state is achieved. We have applied a Monte Carlo method based on the density-matrix equations in the dressed-state representation to simulate the atomic motion, which is applicable to $p \sim p_{c}$. The data for various laser detunings are in good agreement with the Monte Carlo simulations. We also compare the data with momentum distributions calculated using the FokkerPlanck equation, and find that this approach fails for some regions of parameter space.

We have calculated the spontaneous emission lifetimes of atoms channeled in the lowest levels of the standingwave potential and modeled the dynamics of channeling with the Monte Carlo method. A more accurate representation of the dynamics will require a fully-quantummechanical calculation. Precise calculations of the rates for loading the quantum states of the channels and the loss rate out of them may lead to more efficient techniques for adiabatically cooling atoms with intense standing waves. 


\section{ACKNOWLEDGMENTS}

This work was partially supported by a National Institute of Standards and Technology Precision Measure- ment Grant, the Texas Advanced Technology Program, the Robert A. Welch Foundation, and the National Science Foundation. R.G.H. received support from the Alfred P. Sloan Foundation and the Shell Foundation.
[1] See, for instance, D. J. Wineland and W. M. Itano, Phys. Rev. A 20, 1521 (1979).

[2] S. Stenholm, Rev. Mod. Phys. 58, 699 (1986), and references therein.

[3] A. P. Kazantsev, Zh. Eksp. Teor. Fiz. 66, 1599 (1974) [Sov. Phys. JETP 39, 784 (1974)].

[4] V. G. Minogin and O. T. Serimaa, Opt. Commun. 30, 373 (1979).

[5] V. G. Minogin and V. S. Letokhov, Laser Light Pressure on Atoms (Gordon and Breach, New York, 1987), and references therein.

[6] J. P. Gordon and A. Ashkin, Phys. Rev. A 21, 1606 (1980).

[7] J. Dalibard and C. Cohen-Tannoudji, J. Opt. Soc. Am. B 2, 1707 (1985).

[8] E. Kyrölä and S. Stenholm, Opt. Commun. 22, 123 (1977); P. R. Berman and J. Ziegler, Phys. Rev. A 15, 2042 (1977).

[9] N. P. Bigelow and M. G. Prentiss, Phys. Rev. Lett. 65, 555 (1990); J. J. Tollett, J. Chen, J. G. Story, N. W. M. Ritchie, C. C. Bradley, and R. G. Hulet, ibid. 65, 559 (1990).

[10] A. Aspect, J. Dalibard, A. Heidmann, C. Salomon, and C. Cohen-Tannoudji, Phys. Rev. Lett. 57, 1688 (1986).

[11] C. E. Tanner, B. P. Masterson, and C. E. Wieman, Opt. Lett. 13, 357 (1988).

[12] M. Prentiss and A. Cable, Phys. Rev. Lett. 62, 1354 (1989).

[13] R. G. DeVoe, Opt. Lett. 16, 1605 (1991).

[14] For a survey of recent work, see special issue of Appl. Phys. B 54, 321 (1992), and references therein.

[15] P. L. Gould, G. A. Ruff, and D. E. Pritchard, Phys. Rev. Lett. 56, 827 (1986), and references therein.

[16] P. L. Gould, P. J. Martin, G. A. Ruff, R. E. Stoner, J.-L. Picqué, and D. E. Pritchard, Phys. Rev. A 43, 585 (1991).

[17] E. Arimondo, H. Lew, and T. Oka, Phys. Rev. Lett. 43, 753 (1979).

[18] M. G. Prentiss and S. Ezekiel, Phys. Rev. Lett. 56, 46
(1986); C. Salomon, J. Dalibard, A. Aspect, H. Metcalf, and C. Cohen-Tannoudji, ibid. 59, 1659 (1987); V. I. Balykin, V. S. Letokhov, Yu. B. Ovchinnikov, A. I. Sidorov, and S. V. Shul'ga, Opt. Lett. 13, 958 (1988).

[19] J. Chen, J. G. Story, J. J. Tollett, and R. G. Hulet, Phys. Rev. Lett. 69, 1344 (1992).

[20] J. Dalibard, A. Heidmann, C. Salomon, A. Aspect, H. Metcalf, and C. Cohen-Tannoudji, in Fundamentals of Quantum Optics II, edited by F. Ehlotzky (SpringerVerlag, Berlin, 1987).

[21] Y. Castin, H. Wallis, and J. Dalibard, J. Opt. Soc. Am. B 6, 2046 (1989).

[22] J. Dalibard, Y. Castin, and K. Mølmer, Phys. Rev. Lett. 68, 580 (1992); R. Dum, P. Zoller, and H. Ritsch, Phys. Rev. A 45, 4879 (1992).

[23] A. P. Kazantsev, D. O. Chudesnikov, and V. P. Yakovlev, Zh. Eksp. Teor. Fiz. 90, 1619 (1986) [Sov. Phys. JETP 63, 951 (1986)].

[24] V. I. Balykin, V. S. Letokhov, V G. Minogin, Yu. V. Rozhdestvensky, and A. I. Sidorov, J. Opt. Soc. Am. B 2, 1776 (1985).

[25] V. S. Letokhov, Pis'ma Zh. Eksp. Teor. Fiz. 7, 348 (1968) [JETP Lett. 7, 272 (1968)].

[26] A. Ashkin, Phys. Rev. Lett. 40, 729 (1978).

[27] P. Verkerk, B. Lounis, C. Salomon, C. Cohen-Tannoudji, J.-Y. Courtois, and G. Grynberg, Phys. Rev. Lett. 68, 3861 (1992); P. S. Jessen, C. Gerz, P. D. Lett, W. D. Phillips, S. L. Rolston, R. J. C. Spreeuw, and C. I. Westbrook, ibid. 69, 49 (1992).

[28] R. J. Cook, Phys. Rev. A 22, 1078 (1980); J. Javanainen and S. Stenholm, Appl. Phys. 21, 35 (1980); 21, 163 (1980); C. Tanguy, S. Reynaud, M. Matsuoka, and C. CohenTannoudji, Opt. Commun. 44, 249 (1983).

[29] H. Risken, The Fokker-Planck Equation, 2nd ed. (Springer-Verlag, Berlin, 1989). 